\title{
Burden of Iron Deficiency Anemia in a Bariatric Surgery Population in the United States
}

\author{
Tyler Knight, MS; Lynell D'Sylva, BSN, RN; Brad Moore, MBA; and Charles F. Barish, MD
}

\begin{abstract}
BACKGROUND: Obesity is a serious condition affecting more than $35 \%$ of adults in the United States. In obese individuals for whom other weight control methods have been ineffective, bariatric surgery is a safe and effective method of weight control. An estimated 150,000 to 160,000 bariatric surgeries are performed in the United States yearly. Iron deficiency anemia is common in patients after bariatric surgery, with incidence rates up to $49 \%$, and may be due to malabsorption of nutrients.
\end{abstract}

OBJECTIVES: To (a) compare the medical resource utilization (MRU) - both medical care and treatment resources-and associated costs in a sample of commercially insured adult bariatric surgery patients with and without iron deficiency anemia (IDA), and (b) describe anti-anemia treatment patterns in those bariatric surgery patients diagnosed with IDA.

METHODS: Using Truven Health MarketScan claims data, bariatric surgery patients were identified by the ICD-9-CM and CPT procedure codes for bariatric surgery and classified by surgery and IDA diagnosis within 2 years of initial surgery. Intravenous (IV) iron treatment was determined by HCPCS codes, prescription oral iron by NDC numbers, and blood transfusions by CPT and ICD-9-CM codes. Clinical, MRU, and economic outcomes for allcause health services were compared between IDA and non-IDA patients. Odds ratios (ORs) and $95 \%$ confidence intervals (Cls) were calculated using logistic regression, controlling for demographic and clinical characteristics on outcomes of complications and hospitalization.

RESULTS: Of the 24,344 bariatric surgery patients analyzed, $11.6 \%$ received an IDA diagnosis 2 years after surgery (average days to diagnosis $=279$ ). Most IDA patients (78.5\%) received a test for iron in the post-index period; only $9.1 \%$ received IV iron treatment, with iron dextran (3.8\%) and iron sucrose $(3.4 \%)$ being the most common (average days to IV iron treatment $=403$ days). Prescription oral iron was found in $4.9 \%$ of all IDA patients (average days to oral iron treatment $=476.7$ ). Approximately $9 \%$ of IDA patients received a blood transfusion (average days to transfusion $=304.8$ ). For the total sample, the average age was 46 years with a higher percentage of females (83.9\% IDA; $78.7 \%$ non-IDA). Most clinical characteristics were similar among patients with and without IDA, except heart disease (1.3\% IDA vs. $0.8 \%$ non-IDA; $P=0.005)$ and gallbladder disease $(0.0 \%$ IDA vs. $0.2 \%$ non-IDA; $P=0.037)$. More IDA patients had complications after surgery (40.4\% vs. $27.7 \%$; $P<0.001)$, such as nonabsorption $(22.4 \%$ vs. $16.5 \%$; $P<0.001)$; digestive (15.6\% vs. $10.2 \% ; P<0.001$ ); and gastrojejunal ulcer (7.6\% vs. $2.0 \%$; $P<0.001)$. Multivariate results showed that IDA patients were more likely to have a bariatric surgery complication over non-IDA patients $(0 \mathrm{R}=1.367,95 \% \mathrm{Cl}=1.257-1.487 ; P<0.05)$. Adjusted results showed IDA patients more than twice as likely to be hospitalized $(0 \mathrm{R}=2.567,95 \%$ $\mathrm{Cl}=2.363-2.790 ; P<0.05)$. Total costs were twice as much in the IDA group compared with the non-IDA group $(\$ 37,882$ vs. $\$ 19,253 ; P<0.001)$.

CONCLUSIONS: Bariatric surgery patients who develop IDA may be subject to higher complication rates, MRU, and direct medical costs. Although most bariatric surgery patients who develop IDA are tested for iron, most are not treated with IV iron or oral iron and do not receive blood transfusions. Further research is needed to determine if IDA is a result of bariatric surgery complications or a predictor of increased MRU and costs.

\section{J Manag Care Spec Pharm. 2015;21(10):946-54}

Copyright $\odot 2015$, Academy of Managed Care Pharmacy. All rights reserved.

\section{What is already known about this subject}

Iron deficiency anemia is a frequent complication in gastric bypass surgery (33\% to $49 \%$ of all gastric bypass patients).

When anemia increases in severity, patients may present with nonspecific symptoms, such as fatigue, pallor, and dyspnea on exertion.

\section{What this study adds}

This study uses real-world evidence to describe the economic impact of iron deficiency anemia on patients with recent bypass surgery. This study also investigates the onset of iron deficiency anemia and average time to treatment, specifically analyzing oral and intravenous iron, no treatment, and blood transfusions.

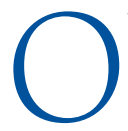
besity is a serious condition affecting over one third (35.7\%) of adults in the United States. ${ }^{1}$ Among other problems, obesity causes an overall increase in the obesity-related disease burden with such comorbidities as type 2 diabetes, gallbladder disease, cardiovascular disease, high cholesterol, high blood pressure, and osteoarthritis. ${ }^{2}$ In cases in which diet, exercise, behavioral modification, and drugs are not effective, bariatric surgery provides a safe, effective alternative for weight control and prevention of associated comorbidities. ${ }^{3}$

Estimates show there were approximately 150,000 to 160,000 cases of bariatric surgery performed in the United States in 2010. ${ }^{4}$ Bariatric surgery results in substantial weight loss and helps prevent, improve, or resolve more than 40 obesity-related diseases or conditions, including type 2 diabetes, heart disease, and certain cancers..$^{5-7}$ Additionally, according to 1 study, gastric bypass may improve a patient's life expectancy by $89 \%$ and reduce risk of premature death by $30 \%$ to $40 \% .8,9$

With the growing prevalence of obesity and associated cost burden, bariatric surgery is generally considered safe because of increased use of laparoscopy (increasing from 20.1\% in 2003 to $90.2 \%$ in 2008), ${ }^{10}$ advancements in surgical techniques, ${ }^{3}$ 
and establishment of the American Society for Metabolic and Bariatric Surgery and American College of Surgeons accreditation programs. ${ }^{11}$ However, data from several sources indicate that complications due to malabsorption are frequently associated with bariatric surgery. ${ }^{12-14}$

Iron deficiency anemia (IDA) is the most common form of anemia that occurs when the body does not have enough available iron, thus, limiting the production of healthy red blood cells..$^{15}$ IDA affects approximately $30 \%$ of the world's population and remains common in the United States, with studies showing that $5 \%$ to $12 \%$ of nonpregnant women and $1 \%$ to $5 \%$ of men have IDA. ${ }^{16}$ The American Society of Hematology notes that people who have undergone bariatric procedures, especially gastric bypass surgery, are among the highest risk for IDA with 33\% to $49 \%$ of patients who undergo gastric bypass surgery experiencing IDA..$^{17,18}$ IDA typically occurs 8 weeks to 2 years after surgery, with deficiencies of iron, folate, and vitamin $\mathrm{B} 12$ being the most commonly reported etiologies. ${ }^{19}$ Patients with mild IDA are most likely asymptomatic; however, when the anemia increases in severity, patients may present with nonspecific symptoms, such as fatigue, pallor, and dyspnea on exertion. ${ }^{16}$

Treatment for anemia typically focuses on increasing iron stores until normal levels are restored. Prescribing iron supplement pills is 1 method of restoring a patient's iron stores; however, some individuals experience adverse effects from oral supplements. Known adverse effects of oral iron supplements include abdominal discomfort, nausea, vomiting, diarrhea, and constipation, which may contribute to noncompliance. A patient may not be capable of absorbing iron via the gastrointestinal tract-a trait commonly observed in gastric bypass patients. In these cases, intravenous (IV) iron may be necessary to treat anemia. In more severe cases, a blood transfusion may be required when a patient is hemodynamically unstable. ${ }^{17}$ The most common IV iron treatments include iron dextran, iron sucrose, and iron gluconate. Ferumoxytol is another IV iron treatment but is indicated specifically for patients with chronic kidney disease (CKD). ${ }^{17}$ All IV iron products carry the risk of causing anaphylactic/anaphylactoid reactions, which can be life threatening. Therefore, a test dose is recommended before each therapeutic dose for iron dextran, and a 30-minute observation period is recommended for iron sucrose, ferric gluconate, ferumoxytol, and ferric carboxymaltose after administration. Allergic reactions are more common with iron dextran and may necessitate switching to a different preparation. ${ }^{16}$ Severe side effects other than allergic reactions are rare and include urticaria, pruritus, and muscle and joint pain.

While it has been established that bariatric surgery presents a safe and effective alternative for weight loss and prevention of obesity-related comorbidities, it also results in a degree of medical resource utilization (MRU) - the use or consumption of resources related to medical care or treatment including, but not limited to, hospitalizations, physician office visits, hospi- tal outpatient visits, and outpatient prescriptions use-and outcomes that are both positive and negative. Multiple studies have evaluated the impact of bariatric surgery and associated MRU and cost; however, these studies did not analyze the effect of IDA diagnosis and treatment on economic variables in this study population..$^{20-23}$

This study aims to compare the MRU and associated costs in a commercially insured bariatric surgery population with and without IDA and to describe anti-anemia treatment patterns in those patients with IDA over a 2-year postsurgery period.

\section{Methods}

\section{Study Design and Data Sources}

This was a retrospective, longitudinal cohort study based on health insurance claims data from a U.S. private-payer data source using the most recent 5-year period (January 1, 2007, through December 31, 2011). The primary analysis used the MarketScan Commercial Claims and Encounters and Medicare Supplemental Databases of Truven Health Analytics.

The MarketScan Commercial Database includes inpatient and outpatient medical claims and outpatient prescription drug claims for over 170 million privately insured individuals in the United States annually. These individuals come from approximately 90 large employers and health plans with insurance provided under various fee-for-service and capitated health plans. ${ }^{24}$

\section{Study Population}

Patients with bariatric surgery were identified by the presence of at least 1 inpatient or outpatient medical claim between January 1, 2008, and December 31, 2009, with International Classification of Diseases, Ninth Revision, Clinical Modification (ICD-9-CM) or Current Procedural Terminology procedure codes for bariatric surgery (restrictive, malabsorptive, and combined restrictive/malabsorptive bariatric surgeries). Eligible bariatric surgery patients must have been at least aged 18 years at the time of surgery and must have shown continuous enrollment in one of the MarketScan health insurance plans 1 year before and 2 years after initial bariatric surgery. Patients with a primary or secondary diagnosis of CKD at any point in the study period were excluded.

The index event for each patient was the date of bariatric surgery. Pre-index and post-index periods were established for each patient that represented the 1-year period before and the 2-year period after the index event, respectively. Patients with anemia were identified by the presence of a primary or secondary diagnosis code for anemia (ICD-9-CM codes 280.x, 285.1, 648.2x, 285.22, 285.29, and 285.3) during the post-index period.

Treatment was investigated for patients with anemia and included IV iron (iron sucrose, iron dextran, iron gluconate, and ferumoxytol) and prescription oral iron. IV iron treatment was determined by Healthcare Common Procedure Coding System codes, and prescription oral iron treatment was determined by National Drug Code numbers. 


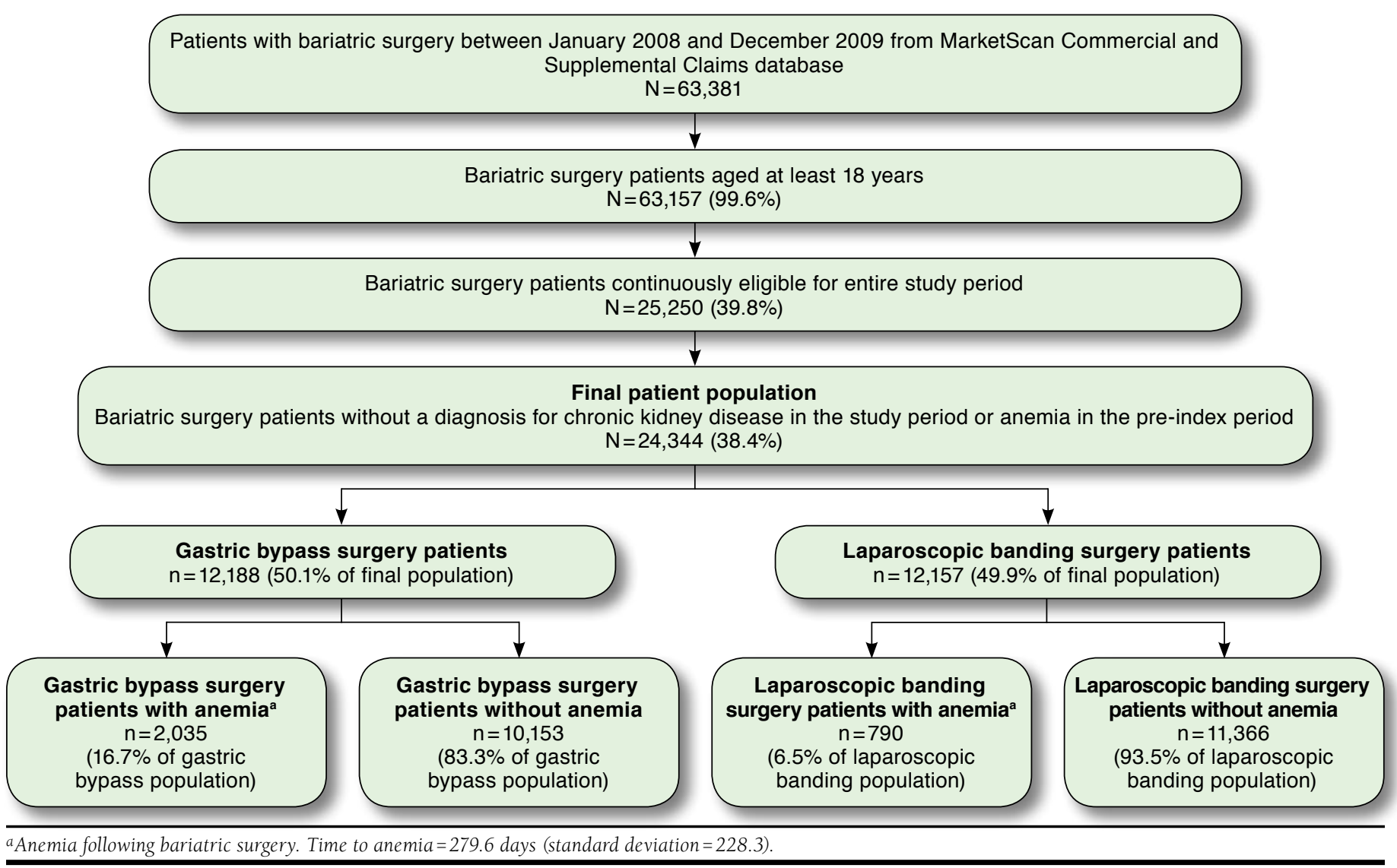

\section{Study Endpoints}

Outcomes, including bariatric surgery complications and allcause hospitalization, were investigated during the post-index period and compared between patients with and without IDA. Bariatric surgery complications included bariatric surgery status complicating pregnancy, childbirth, or the puerperium (ICD9-CM 649.2); digestive system complications (ICD-9-CM 997.4); infection and other complications due to other bariatric procedure (ICD-9-CM 539.81); other complications of other bariatric procedure (ICD-9-CM 539.89); other and unspecified postsurgical nonabsorption (ICD-9-CM 579.3); pica (ICD-9-CM 307.52); postgastric surgery syndrome (ICD-9-CM 564.2); gastrojejunal ulcer (ICD-9-CM 534); and hernia of other specified sites with gangrene (ICD-9-CM 551.8 and 552.8). Costs associated with all-cause MRU were derived from medical and pharmacy claims paid by the insurer and were reported across settings: hospital inpatient, hospital outpatient (hospital-based outpatient department), physician office, emergency room, and gastroenterologist facility. Outpatient prescription costs were reported. Costs were summarized over the 2-year post-index period and adjusted to the most recent year of data in the MarketScan database (2012) by the medical component of the Consumer Price Index.

\section{Analysis Methods}

Descriptive statistics were used to summarize demographic and clinical characteristics, outcomes, MRU, and costs. Categorical variables were presented as the count and percentage of patients in each category, and chi-square tests were used to compare differences between groups. Continuous variables were summarized using mean, standard deviation, median, and range, as appropriate. Kruskal-Wallis tests were used to compare costs between groups, and t-tests were used to test all other continuous variables between groups. Odds ratios (ORs) and 95\% confidence intervals (CIs) were calculated using logistic regression controlling for demographic and clinical characteristics (including post-index anemia status, diabetes, cancer, sleep apnea, heart disease, stroke, hypertension, dyslipidemia, gallbladder disease, osteoarthritis, and chronic back pain) on the outcomes of bariatric surgery complications and hospitalization in the post-index period. All statistical analyses were performed using SAS version 9.1.3 (SAS Institute, Cary, NC).

\section{Results}

At the start of the study, patients who had bariatric surgery between January 2008 and December 2009 were determined 
Burden of Iron Deficiency Anemia in a Bariatric Surgery Population in the United States

TABLE 1 Demographic and Clinical Characteristics of Study Population

\begin{tabular}{|c|c|c|c|c|c|c|c|}
\hline & \multicolumn{2}{|c|}{$\begin{array}{c}\text { Overall } \\
(\mathrm{N}=24,344)\end{array}$} & \multicolumn{2}{|c|}{$\begin{array}{l}\text { Anemia }^{\mathrm{a}} \\
(\mathrm{n}=2,825)\end{array}$} & \multicolumn{2}{|c|}{$\begin{array}{l}\text { No Anemia } \\
(\mathrm{n}=21,519)\end{array}$} & $P$ Value ${ }^{b}$ \\
\hline \multicolumn{8}{|l|}{ Demographics } \\
\hline Age, mean (SD) & 45.9 & $(10.6)$ & 46.3 & $(10.5)$ & 45.9 & $(10.6)$ & 0.0279 \\
\hline Sex, n (\%) & & & & & & & $<0.0001$ \\
\hline Male & 5,030 & $(20.7)$ & 456 & $(16.1)$ & 4,574 & $(21.3)$ & \\
\hline Female & 19,314 & $(79.3)$ & 2,369 & $(83.9)$ & 16,945 & $(78.7)$ & \\
\hline \multicolumn{8}{|l|}{ Surgery type, n (\%) } \\
\hline Gastric bypass & 12,188 & $(50.1)$ & 2,035 & $(72.0)$ & 10,153 & $(47.2)$ & $<0.0001$ \\
\hline Laparoscopic gastric banding & 12,156 & $(49.9)$ & 790 & $(28.0)$ & 11,366 & $(52.8)$ & $<0.0001$ \\
\hline \multicolumn{8}{|c|}{ Clinical characteristics in pre-index period, $\mathbf{n}(\%)$} \\
\hline Anemia & 0 & $(0.0)$ & 0 & $(0.0)$ & 0 & $(0.0)$ & NA \\
\hline Obesity & 1,512 & $(6.2)$ & 181 & $(6.4)$ & 1,331 & $(6.2)$ & 0.6460 \\
\hline Diabetes & 1,151 & $(4.7)$ & 155 & $(5.5)$ & 996 & $(4.6)$ & 0.0433 \\
\hline Cancer & 276 & $(1.1)$ & 34 & $(1.2)$ & 242 & $(1.1)$ & 0.7094 \\
\hline Sleep apnea & 802 & $(3.3)$ & 89 & $(3.2)$ & 713 & $(3.3)$ & 0.6483 \\
\hline Heart disease & 208 & $(0.9)$ & 37 & (1.3) & 171 & $(0.8)$ & 0.0052 \\
\hline Stroke & 12 & $(0.0)$ & 1 & $(0.0)$ & 11 & $(0.1)$ & 0.7234 \\
\hline Hypertension & 1,316 & (5.4) & 163 & (5.8) & 1,153 & (5.4) & 0.3628 \\
\hline Dyslipidemia & 378 & (1.6) & 44 & (1.6) & 334 & (1.6) & 0.9826 \\
\hline Gallbladder disease & 33 & $(0.1)$ & 0 & $(0.0)$ & 33 & $(0.2)$ & 0.0373 \\
\hline Osteoarthritis & 277 & (1.1) & 37 & (1.3) & 240 & (1.1) & 0.3596 \\
\hline Chronic back pain & 364 & (1.5) & 51 & (1.8) & 313 & (1.5) & 0.1486 \\
\hline
\end{tabular}

a Anemia following bariatric surgery.

bStudent's t-test for continuous variables and the Pearson chi-square test for categorical variables.

$N A=$ not applicable; $S D=$ standard deviation .

based on the MarketScan Commercial and Supplemental Claims database $(N=63,381)$. After applying eligibility parameters, the final patient population (i.e., bariatric surgery patients without a diagnosis for CKD in the study period or anemia in the pre-index period) consisted of 24,344 patients. The 2 resulting patient groups were gastric bypass surgery patients $(\mathrm{n}=12,188)$ and laparoscopic banding surgery patients $(\mathrm{n}=12,157)$. Of the gastric bypass surgery patients, there were $16.7 \%$ with anemia $(n=2,035)$ and $83.3 \%$ without anemia $(n=10,153)$. Of the laparoscopic banding surgery patients, there were $6.5 \%$ with anemia $(n=790)$ and $93.5 \%$ without anemia $(n=11,366)$. A complete description of the study population and inclusion/exclusion steps are included in Figure 1.

The mean age of the overall patient population of 24,344 was approximately 46 years and was consistent across the groups with and without anemia. Both patient groups had a higher distribution of females over males. In the anemia group, $83.9 \%$ were female, while $78.7 \%$ were female in the group without anemia. A significant difference $(P<0.001)$ was noted in the proportions of patients with anemia with laparoscopic banding surgery compared with gastric bypass surgery. Conditions with the highest prevalence within the overall study population included obesity $(n=1,512)$, hypertension $(n=1,316)$, diabetes $(n=1,151)$, and sleep apnea $(n=802)$. Clinical characteristics observed with $P$ values $<0.05$ included heart disease $(P=0.005)$ and gallbladder disease $(P=0.037)$. A detailed list of clinical characteristics in the pre-index period is included in Table 1.

The description of all anemia treatments and tests is detailed in Table 2. The average time (days) observed until anemia diagnosis was 279 days (9 to 10 months) in the anemia patient population. Most (78.5\%) anemia patients received a test for iron in the post-index period, and $9.1 \%$ of all anemia patients received IV iron treatment; iron dextran (3.8\%) and iron sucrose $(3.4 \%)$ were the most common treatments (average days until IV iron treatment $=406$ days). Prescription oral iron was used in $4.9 \%$ of all anemia patients (average days to oral iron treatment $=476.7$ days). Approximately 9\% of all anemia patients received a blood transfusion, and the average time (days) until blood transfusion was approximately 304.8 days.

In an analysis of bariatric surgery complications (Figure 2), the overall complication rate was observed at $40.4 \%$ for the group with anemia and $27.7 \%$ for the group without anemia. There were particularly high rates of digestive system complications, other and unspecified postsurgical nonabsorption, and intestinal malabsorption observed. A significant difference $(P<0.001)$ was observed in gastrojejunal ulcer rates between the 2 groups with the anemia group associated with a rate 3 times higher than the group without anemia. 
Burden of Iron Deficiency Anemia in a Bariatric Surgery Population in the United States

TABLE 2 Treatment and Tests for Anemia in Study Population

\begin{tabular}{|c|c|c|c|c|c|c|c|c|c|}
\hline \multirow[b]{2}{*}{ Treatment/Test } & \multirow{2}{*}{\multicolumn{2}{|c|}{ n $(\%)^{a}$}} & \multicolumn{3}{|c|}{ Number of Treatments/Tests } & \multicolumn{4}{|c|}{ Time to Treatment (Days) } \\
\hline & & & \multicolumn{2}{|c|}{ Mean (SD) } & Median (Range) & \multicolumn{2}{|c|}{ Mean (SD) } & \multicolumn{2}{|c|}{ Median (Range) } \\
\hline \multicolumn{10}{|l|}{ Anemia treatments } \\
\hline Any IV iron & 258 & $(9.1)$ & 0.4 & $(1.9)$ & $0.00 \quad(0.0-30.0)$ & 406.0 & $(214.9)$ & 435.00 & $(0.0-728.0)$ \\
\hline Iron sucrose & 96 & (3.4) & 0.2 & (1.3) & $0.00 \quad(0.0-28.0)$ & 402.6 & $(231.5)$ & 452.50 & $(0.0-730.0)$ \\
\hline Iron dextran & 106 & (3.8) & 0.1 & $(0.9)$ & $0.00 \quad(0.0-24.0)$ & 403.2 & (203.8) & 421.00 & $(0.0-714.0)$ \\
\hline Iron gluconate & 53 & (1.9) & 0.1 & $(1.0)$ & $0.00 \quad(0.0-26.0)$ & 402.4 & $(220.1)$ & 414.00 & $(7.0-714.0)$ \\
\hline Ferumoxytol & 18 & $(0.6)$ & 0.0 & $(0.3)$ & $0.00 \quad(0.0-10.0)$ & 536.9 & $(127.8)$ & 567.00 & 249.0-728.0) \\
\hline Oral iron & 138 & $(4.9)$ & 0.2 & $(1.6)$ & $0.00 \quad(0.0-25.0)$ & 476.7 & $(229.8)$ & 552.50 & $(2.0-730.0)$ \\
\hline Blood transfusion & 256 & (9.1) & 0.1 & $(0.5)$ & $0.00 \quad(0.0-15.0)$ & 304.8 & $(253.6)$ & 308.50 & $(0.0-730.0)$ \\
\hline \multicolumn{10}{|l|}{ Iron tests } \\
\hline Ferritin laboratory test & 1,708 & $(60.5)$ & 1.6 & $(2.0)$ & $1.00 \quad(0.0-20.0)$ & \multicolumn{2}{|c|}{ NA } & \multicolumn{2}{|c|}{ NA } \\
\hline Transferrin laboratory test & 294 & $(10.4)$ & 0.2 & $(0.8)$ & $0.00 \quad(0.0-10.0)$ & \multicolumn{2}{|c|}{ NA } & \multicolumn{2}{|r|}{ NA } \\
\hline Iron laboratory test & 1,993 & $(70.5)$ & 2.0 & $(2.1)$ & $1.00 \quad(0.0-15.0)$ & \multicolumn{2}{|c|}{ NA } & \multicolumn{2}{|c|}{ NA } \\
\hline Iron capacity binding laboratory test & 1,577 & $(55.8)$ & 1.4 & $(1.8)$ & $1.00 \quad(0.0-14.0)$ & \multicolumn{2}{|c|}{ NA } & \multicolumn{2}{|r|}{ NA } \\
\hline Any iron test & 2,218 & $(78.5)$ & 5.2 & $(5.4)$ & $4.00 \quad(0.0-42.0)$ & \multicolumn{2}{|c|}{ NA } & \multicolumn{2}{|c|}{ NA } \\
\hline
\end{tabular}

a Based on patients with anemia following bariatric surgery $(N=2,825)$.

$I V=$ intravenous; $N A=$ not applicable, $S D=$ standard deviation.

In the all-cause post-index MRU observations (Table 3), it was noted that the anemia group had nearly twice the number of hospitalizations (42.8\%) than the group without anemia (20.9\%). Among those hospitalized, patients in the anemia group were in the hospital longer compared with patients in the group without anemia—over 3 days longer on average. Patient visits to the gastroenterologist were nearly double among patients with anemia compared with patients without anemia (22.8\% vs. $12.6 \%$, respectively). Proportions of patients with hospital outpatient visits and physician office visits were slightly higher in the anemia group. More emergency room visits were also observed in the anemia group (43.0\%) compared with the group without anemia (34.4\%). While the number of patients with outpatient prescription use was similar, anemia patients had approximately 10 more prescriptions per person compared with patients without anemia over the 2-year follow-up period.

In a multivariate logistic regression analysis of outcomes (Table 4), anemia patients were more than twice as likely to be hospitalized during the post-index period $(\mathrm{OR}=2.567,95 \%$ $\mathrm{CI}=2.363-2.790 ; P<0.05)$. In addition, anemia patients were nearly $37 \%$ more likely to have had a bariatric surgery complication in the post-index period $(\mathrm{OR}=1.367,95 \% \mathrm{CI}=1.257$ 1.487; P<0.05).

Finally, the all-cause post-index MRU within the overall study population is detailed in Figure 3. Total MRU 2-year private payer reimbursement cost (adjusted for 2012 U.S. dollars) was nearly double in the anemia group compared with the group without anemia ( $\$ 37,882$ and $\$ 19,253$ respectively). Further cost breakdown of MRU by setting of care includes hospitalizations, hospital physician outpatient, physician office, gastroenterologist, emergency room, outpatient prescriptions, and other.

\section{Discussion}

There has been a consistent, widespread increase in the prevalence and incidence of obesity in the U.S. population. Because of this trend, bariatric surgery has emerged as a safe, effective alternative for weight control, particularly in cases where diet, exercise, and behavior modifications have not been successful. Patients who undergo bariatric surgery are susceptible to IDA. As with any health condition, once a patient develops IDA, the resulting requirements for care and treatment lead to increased MRU and associated costs. Multiple studies have reported the correlation between IDA and increased direct and indirect cost burden. ${ }^{25-27}$ For example, in the study by Nissenson et al. (2005), the investigators concluded that medical costs for anemic patients were twice as much as those for nonanemic patients with the same comorbid condition. ${ }^{27}$ These findings are similar to those in this study that focuses on postbariatric surgery patients.

Based on observations of the study population, it was noted that most patients had some type of iron test results, and it appeared that gastric bypass patients were being monitored for IDA. However, $11.7 \%$ of the study sample received a diagnosis of IDA that is significantly lower than the 33\% to $49 \%$ of patients who undergo gastric bypass surgery and then experience IDA that has been reported in the literature. ${ }^{18}$ This could infer that IDA was underdiagnosed in this sample; IDA may be reported under an unspecified anemia ICD-9-CM diagnosis code such as 285.9; or that the incidence was simply low in this study.

By nature, bariatric surgery patients tend to have malabsorptive issues and are unable to use oral iron supplementation. Therefore, it can be expected that a higher proportion of patients would have received either IV iron infusions or a blood 


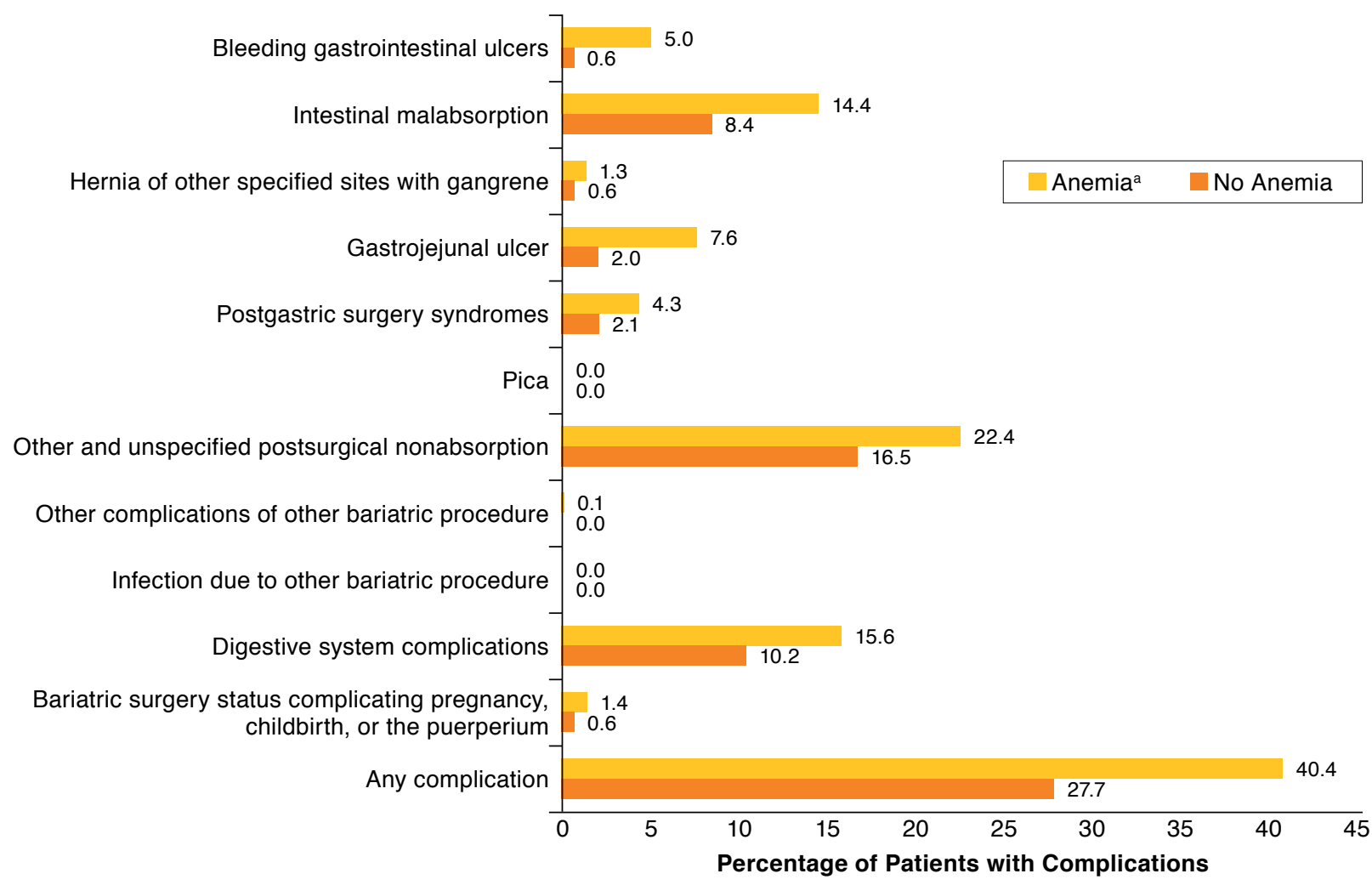

Note: No patients had an infection due to other bariatric procedures. With the exception of infection, "infection due to other bariatric procedure" where neither group reported this complication, comparisons of complications between patients with and without anemia were all significant $(P<0.05)$.

aAnemia following bariatric surgery.

transfusion. However, the data showed that only a small percentage of the monitored patients actually received either IV iron or blood transfusion treatment (9.0\% and $9.3 \%$, respectively). In a recent study of Medicare beneficiaries with stage 3 or 4 CKD and anemia, anti-anemia therapy was utilized in roughly $36 \%$ of the study sample (413 out of 3,897 ), and the investigators found that patients not treated with IV iron had an increased cost compared with cohorts treated with IV iron. ${ }^{28}$ Although CKD patients were excluded from the present study, findings are similar to the small percentage of postbariatric surgery patients receiving anemia management therapy, which may help to reduce costs; however, an analysis of costs between anemic patients receiving anti-anemia therapy compared with those not receiving antianemia therapy was not conducted and may be a topic for further investigation. Furthermore, the reasons for the underutilization of IV iron therapy, which may include delaying treatment until symptoms become more severe and/or concerns related to lack of benefit or side effects, are another topic of investigation in the postbariatric surgery population.
The most significant contributors to medical costs in both groups were hospitalizations and hospital outpatient visits, collectively comprising $71 \%$ and $62 \%$ in the anemia and nonanemia groups, respectively. The anemia group had a higher rate of MRU overall and, therefore, higher costs. For example, the anemia group had nearly twice the number of hospitalizations with an average length of stay that was 3 days longer than the group without anemia during those hospitalizations (8.3 vs. 5.2 days). The resulting 2-year cumulative hospitalization cost in the anemia group was over 3 times higher compared with the hospitalization cost in the group without anemia, and this was the largest difference between the 2 groups. While not as substantial of a difference, the trend was consistent across nearly all of the utilization parameters. The only parameter with similar proportions was outpatient prescriptions (98\% in both patient groups), but it should be noted that anemic patients had approximately 10 more outpatient prescriptions on average over the 2-year follow-up period.

The anemia group was associated with more bariatric surgery complication outcomes compared with the group without 
TABLE 3 All-Cause Post-Indexa Medical Resource Use of Study Population

\begin{tabular}{|c|c|c|c|c|}
\hline Setting of Care & $\begin{array}{c}\text { Overall } \\
(\mathrm{N}=24,344) \\
\end{array}$ & $\begin{array}{c}\text { Anemiab } \\
(\mathrm{n}=2,825)\end{array}$ & $\begin{array}{l}\text { No Anemia } \\
(\mathrm{n}=21,519)\end{array}$ & $P$ Value $^{\mathrm{c}}$ \\
\hline \multicolumn{5}{|l|}{ Hospitalizations } \\
\hline $\mathrm{n}(\%)$ & $(23.5)$ & $(42.8)$ & $(20.9)$ & $<0.0001$ \\
\hline Mean (SD) & $(0.9)$ & $(1.4)$ & $(0.7)$ & $<0.0001$ \\
\hline Median (range) & $0.0 \quad(0.0-22.0)$ & $0.0 \quad(0.0-22.0)$ & $0.0 \quad(0.0-15.0)$ & \\
\hline Total LOSd & & & & $<0.0001$ \\
\hline Number & 5,717 & 1,210 & 4,507 & \\
\hline Mean (SD) & $(10.7)$ & $(14.5)$ & (9.4) & $<0.0001$ \\
\hline Median (range) & $3.0(1.0-279.0)$ & $4.0(1.0-185.0)$ & $3.0(1.0-279.0)$ & \\
\hline \multicolumn{5}{|l|}{ Hospital outpatient visits } \\
\hline $\mathrm{n}(\%)$ & 22,127 & $(95.7)$ & 19,424 & $<0.0001$ \\
\hline Mean (SD) & $(9.4)$ & $(13.7)$ & $(8.6)$ & $<0.0001$ \\
\hline Median (range) & $5.0(0.0-190.0)$ & $7.0(0.0-190.0)$ & $5.0(0.0-152.0)$ & \\
\hline \multicolumn{5}{|l|}{ Outpatient physician office visits } \\
\hline $\mathrm{n}(\%)$ & 24,153 & $(99.6)$ & 21,340 & 0.0211 \\
\hline Mean (SD) & $(22.4)$ & $(24.5)$ & $(22.0)$ & $<0.0001$ \\
\hline Median (range) & $19.0(0.0-607.0)$ & $24.0(0.0-250.0)$ & $19.0(0.0-607.0)$ & \\
\hline \multicolumn{5}{|l|}{ Gastroenterologist visits } \\
\hline $\mathrm{n}(\%)$ & $(13.8)$ & $(22.8)$ & $(12.6)$ & $<0.0001$ \\
\hline Mean (SD) & $(1.2)$ & $(1.8)$ & $(1.0)$ & $<0.0001$ \\
\hline Median (range) & $0.0 \quad(0.0-35.0)$ & $0.0 \quad(0.0-28.0)$ & $0.0 \quad(0.0-35.0)$ & \\
\hline \multicolumn{5}{|l|}{ Emergency room visits } \\
\hline $\mathrm{n}(\%)$ & $(35.4)$ & $(43.0)$ & $(34.4)$ & $<0.0001$ \\
\hline Mean (SD) & $(1.7)$ & $(2.6)$ & $(1.6)$ & $<0.0001$ \\
\hline Median (range) & $0.0 \quad(0.0-58.0)$ & $0.0 \quad(0.0-57.0)$ & $0.0 \quad(0.0-58.0)$ & \\
\hline \multicolumn{5}{|l|}{ Outpatient prescription claims } \\
\hline $\mathrm{n}(\%)$ & 23,746 & 2,766 & 20,980 & 0.1790 \\
\hline Mean (SD) & $(43.5)$ & $(48.7)$ & $(42.6)$ & $<0.0001$ \\
\hline Median (range) & $33.0(0.0-467.0)$ & $41.0(0.0-419.0)$ & $32.0(0.0-467.0)$ & \\
\hline \multicolumn{5}{|c|}{$\begin{array}{l}\text { aExcludes initial bariatric surgery. } \\
\text { bAnemia following bariatric surgery. } \\
\text { cStudent's t-test for continuous variables and the Pearson chi-square test for categorical variables. } \\
\text { dAmong patients with a hospitalization. } \\
\text { LOS=length of stay; SD=standard deviation. }\end{array}$} \\
\hline
\end{tabular}

anemia for every complication that was measured $(P<0.05$ for all pairwise comparisons between groups). The most commonly reported complications in both groups included other and unspecified postsurgical nonabsorption, digestive system complications, and intestinal malabsorption. After controlling for demographics and clinical characteristics, anemia patients were still at more than twice the risk as patients without anemia to have had a postsurgical complication and about 37\% more likely to be hospitalized in the post-index period. It is not surprising that anemic patients had higher postsurgical complications measured in this study, since it is well documented and widely accepted that nonabsorption of key vitamins and iron is common after bariatric surgery and often leads to IDA. In milder cases, IDA does not usually cause complications; however, if left untreated, the potential for other systemic complications is substantially higher. ${ }^{29}$ This increased potential for complications implies that these patients are more likely to be hospitalized after surgery and require consistent monitoring and treatment regimens.
TABLE 4 Multivariate Logistic Regression for Outcomes of Hospitalization and Bariatric Surgery Complication as a Function of Demographic and Baseline Clinical Characteristics ${ }^{\mathrm{a}}$

\begin{tabular}{|c|c|c|}
\hline Outcome & $\begin{array}{l}\text { Adjusted Odds Ratiob } \\
\text { Patients with Anemia }\end{array}$ & C-statistic \\
\hline Hospitalization & $2.567(2.363-2.790)^{c}$ & 0.612 \\
\hline Bariatric surgery complications (any) & $1.367(1.257-1.487)^{c}$ & 0.658 \\
\hline \multicolumn{3}{|c|}{$\begin{array}{l}\text { Note: Initial surgery not included in hospitalization outcome. } \\
\text { aBaseline clinical characteristics include history (1 year before index quarter) of } \\
\text { anemia, obesity, diabetes, cancer, sleep apnea, heart disease, stroke, hypertension, } \\
\text { dyslipidemia, gallbladder disease, osteoarthritis, and chronic back pain. } \\
\text { bReference group is patients without anemia after bariatric surgery. } \\
\text { cIndicates that the associated } P \text { value is less than } 0.05 \text {. }\end{array}$} \\
\hline
\end{tabular}

\section{Limitations}

It is important to note the limitations of the analysis method used for this study. The observations of this study are based only 


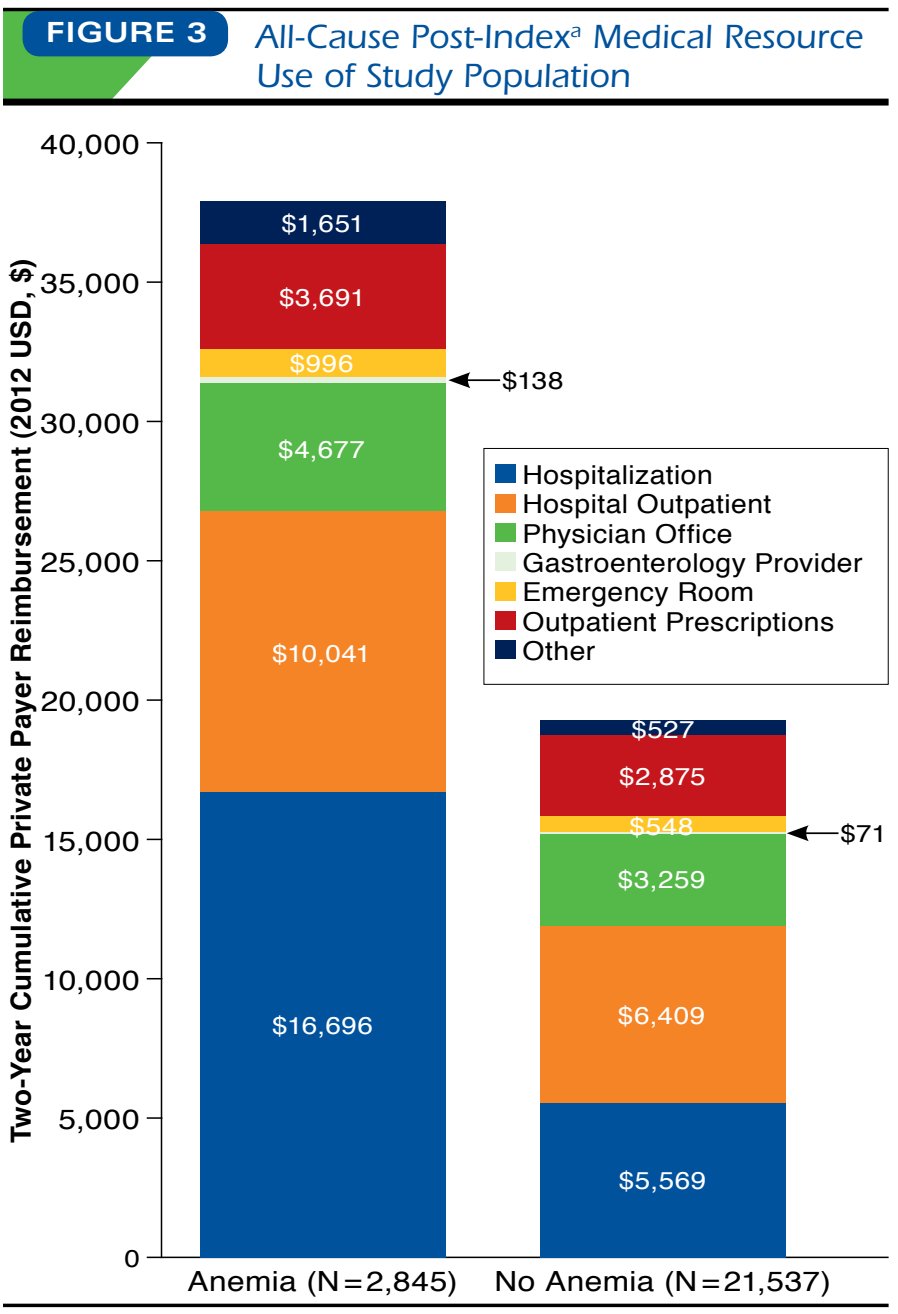

Note: Gastroenterology provider accounted for $\$ 138$ in the anemia group and $\$ 71$ in the no anemia group. Kruskal-Wallis tests were conducted to assess differences in costs by setting of care between patients with and without anemia. All comparisons showed significant differences $(P<0.05)$.

${ }^{a}$ Excludes initial bariatric surgery.

USD = U.S. dollars

on patients who sought health care with full commercial insurance coverage and as a result would be reflected in the health system. Bariatric surgery patients who did not seek health care could not be included; therefore, costs and resource use may not be properly represented. In terms of study targets, several indirect costs were not measured, such as impact on productivity, lost wages, and caregiver support. Another limitation of the analysis is that the retrospective nature does not allow for the attribution of causality of outcomes. However, data from retrospective database studies can indicate associations worthy of further investigation using additional research designs. Because the goal of this analysis was to evaluate costs from a societal perspective, payer reimbursement was used to estimate costs.
An inherent limitation of administrative claims analyses is the reliance on the accuracy of coding in the claims. Because anemia is unlikely to be the primary diagnosis on a medical claim for these patients and because the patient may have more serious comorbidities, anemia may not be recorded as a secondary diagnosis. As a result, some anemic patients may have been misclassified and, thus, not included in the anemia cohort for this analysis. On the same note, there is a possibility of overlap among patients who received various forms of irontransfusion, IV iron, or oral iron-and a possibility of mutually exclusive sets of patients. Also, we note that an anemia diagnosis may be required to receive reimbursement for the administration of IV iron treatment; this gives us confidence that few anemia patients were missed in the analysis. At the same time, if anemia diagnoses are being recorded to ensure reimbursement for the administration of IV iron, the sample may be biased to include patients receiving IV iron treatment.

Future considerations for research include a more specific study to determine if IDA is a direct result of bariatric surgery complications or a predictor of increased MRU and costs. Also, past research findings indicate that patients who have a history of obesity and eating disorders have a tendency to compensate with poor lifestyle choices, such as gambling and alcoholism. ${ }^{30,31}$ A potential future analysis involving MRU and associated costs of postbariatric surgery patients with and without IDA that takes patient lifestyle choices into consideration can further emphasize the real-world nature of the data.

\section{Conclusions}

While the economic burdens of IDA and gastric bypass surgery have been widely reported and understood independently, literature that reports real-world data regarding the burden of IDA specific to the gastric bypass surgery patient population has been limited. By performing a retrospective claims analysis, we have explored real-world results on MRU and costs associated with IDA in a gastric bypass surgery population from a private-payer perspective in the United States. Our analysis showed that bariatric surgery patients with anemia were associated with significantly higher MRU and resulting cost compared with the nonanemia population. While there is a clear trend in published literature on increased MRU and costs with IDA and bariatric surgery independently, this study provided additional insight into what happens to patients after bariatric surgery, by IDA status (i.e., those with IDA and those without), using real-world data. With the consistent increase in obesity becoming prevalent globally, it can be expected that the use of procedures such as bariatric surgery will also rise. ${ }^{32-34} \mathrm{It}$ is important to develop a better understanding of the potential impact beyond obesity-related benefits, particularly in the management of these patients after surgery, to develop more effective procedures for the future and prevent an overwhelming increase in MRU and costs. 


\section{Authors}

TYLER KNIGHT, MS, is Associate Director, and BRAD MOORE, MBA, is Manager, Health Economics and Outcomes Research, Covance Market Access Services, Gaithersburg, Maryland. LYNELL D'SYLVA, BSN, RN, is Associate Director of Marketing, American Regent, Shirley, New York, and CHARLES F. BARISH, MD, is Founder and Principal Investigator, Wake Research Associates, Raleigh, North Carolina.

AUTHOR CORRESPONDENCE: Tyler Knight, MS, Covance Market Access Services, 9801 Washingtonian Blvd., 9th Fl., Gaithersburg, MD 20878. Tel.: 240.632.3334; Fax: 240.632.3335;

E-mail: tyler.knight@covance.com.

\section{DISCLOSURES}

American Regent sponsored this research. Covance received funding for this work. Authors are employees of American Regent and Covance based on their roles in study design, analysis and interpretation of data, writing and revising the manuscript, and the decision to submit the manuscript for publication. Knight and Moore are employees of Covance who received funding for this research. D'Sylva is an employee of American Regent. Barish is a paid consultant and was not financially compensated for collaborative efforts on publication-related activities.

Study concept and design were primarily contributed by Knight, along with D'Sylva. Knight and Moore collaborated in the acquisition of data, and Knight, D'Sylva, and Barish interpreted the data. The manuscript was written and revised primarily by Knight, along with D'Sylva and Barish.

\section{ACKNOWLEDGMENTS}

The authors take full responsibility for the content of and the decision to submit this manuscript but thank Teresa A. Oblak, PhD, CMPP; Antonio Ruiz de Esparza of Covance Market Access Services; and Lalitha Chandrashekhar for providing research and editorial support.

\section{REFERENCES}

1. Ogden CL, Carroll MD, Kit BK, Flegal KM. Prevalence of obesity in the United States, 2009-2010. NCHS Data Brief. 2012(82):1-8

2. Must A, Spadano J, Coakley EH, Field AE, Colditz G, Dietz WH The disease burden associated with overweight and obesity. JAMA. 1999;282(16):1523-29

3. Pories WJ. Bariatric surgery: risks and rewards. J Clin Endocrinol Metab. 2008;93(11 Suppl 1):S89-96.

4. American Society for Metabolic and Bariatric Surgery. Fact sheet: metabolic and bariatric surgery. Updated November 2013. Available at: http://s3.amazonaws.com/publicASMBS/MetabolicBariatric SurgeryFactSheetASMBS2013.pdf. Accessed September 5, 2015. 5. Chikunguwo S, Dodson PW, Meador JG, et al. PL-101: Durable resolution of diabetes after Roux-en-Y gastric bypass is associated with maintenance of weight loss. Surg Obes Relat Dis. 2009;5(3 Suppl):S1.

6. Torquati A, Wright K, Melvin W, Richards W. Effect of gastric bypass operation on Framingham and actual risk of cardiovascular events in class II to III obesity. J Am Coll Surg. 2007;204(5):776-82; discussion 782-73.

7. Kaplan LM. Body weight regulation and obesity. J Gastrointest Surg. 2003;7(4):443-51

8. Sjöström L, Narbro K, Sjöström CD, et al. Effects of bariatric surgery on mortality in Swedish obese subjects. N Engl J Med. 2007;357(8):741-52.

9. Adams TD, Gress RE, Smith SC, et al. Long-term mortality after gastric bypass surgery. N Engl J Med. 2007;357(8):753-61.

10. Encinosa WE, Bernard DM, Du D, Steiner CA. Recent improvements in bariatric surgery outcomes. Med Care. 2009;47(5):531-35.

11. American College of Surgeons. Unified national accreditation program for bariatric surgery centers announced by American College of Surgeons and American Society for Metabolic and Bariatric Surgery. March 9, 2011.

Available at: https://www.facs.org/media/press-releases/2012/acs-asmbs0312. Accessed September 5, 2015
12. Malinowski SS. Nutritional and metabolic complications of bariatric surgery. Am J Med Sci. 2006;331(4):219-25.

13. Hammer HF. Medical complications of bariatric surgery: focus on malabsorption and dumping syndrome. Dig Dis. 2012;30(2):182-86.

14. American Society for Metabolic and Bariatric Surgery. Bariatric surgery: postoperative concerns. Revised February 2008. Available at: https://asmbs.org/ resources/bariatric-surgery-postoperative-concerns-2. Accessed September 5, 2015. 15. Medline Plus. Iron deficiency anemia. Updated February 24, 2014. Available at: http://www.nlm.nih.gov/medlineplus/ency/article/000584.htm. Accessed September 5, 2015.

16. Zhu A, Kaneshiro M, Kaunitz JD. Evaluation and treatment of iron deficiency anemia: a gastroenterological perspective. Dig Dis Sci. 2010;55(3):548-59. 17. American Society of Hematology. Iron-deficiency anemia. Available at: http://www.hematology.org/patients/blood-disorders/anemia/5263.aspx. Accessed September 5, 2015.

18. Mizon C, Ruz M, Csendes A, et al. Persistent anemia after Roux-en-Y gastric bypass. Nutrition. 2007;23(3):277-80.

19. Marinella MA. Anemia following Roux-en-Y surgery for morbid obesity: a review. South Med J. 2008;101(10):1024-31.

20. Zhao Y, Encinosa WE. Bariatric surgery utilization and outcomes in 1998 and 2004. Statistical Brief \#23. Healthcare Cost and Utilization Project (HCUP) Statistical Briefs. January 2007. Agency for Healthcare Research and Quality. Rockville, MD. Available at: http://www.ncbi.nlm.nih.gov/books/ NBK63485/. Accessed September 5, 2015.

21. Nguyen NT, Paya M, Stevens CM, Mavandadi S, Zainabadi K, Wilson SE. The relationship between hospital volume and outcome in bariatric surgery at academic medical centers. Ann Surg. 2004;240(4):586-93; discussion 593-84.

22. Gallagher SF, Banasiak M, Gonzalvo JP, et al. The impact of bariatric surgery on the Veterans Administration healthcare system: a cost analysis. Obes Surg. 2003;13(2):245-48.

23. Pope GD, Birkmeyer JD, Finlayson SR. National trends in utilization and inhospital outcomes of bariatric surgery. J Gastrointest Surg. 2002;6(6):855-60; discussion 861.

24. Truven Health Analytics. Databases and online tools. Available at: http:// truvenhealth.com/your-healthcare-focus/life-sciences/data-databases-andonline-tools. Accessed September 5, 2015.

25. Haas JD, Brownlie T 4th. Iron deficiency and reduced work capacity: a critical review of the research to determine a causal relationship. J Nutr. 2001;131(2S-2):676S-688S; discussion 688S-690S.

26. Hunt JM. Reversing productivity losses from iron deficiency: the economic case. J Nutr. 2002;132(4 Suppl):794S-801S.

27. Nissenson AR, Wade S, Goodnough T, Knight K, Dubois RW.

Economic burden of anemia in an insured population. J Manag Care Pharm. 2005;11(7):565-74. Available at: http://www.amcp.org/data/jmcp/Original\%20 Research_565_574.pdf.

28. Knight TG, Ryan K, Schaefer CP, D'Sylva L, Durden ED. Clinical and economic outcomes in Medicare beneficiaries with stage 3 or stage 4 chronic kidney disease and anemia: the role of intravenous iron therapy. J Manag Care Pharm. 2010;16(8):605-15. Available at: http://www.amcp.org/data/jmcp/605-615.pdf. 29. Mayo Clinic. Iron deficiency anemia complications. January 2, 2014. Available at: http://www.mayoclinic.com/health/iron-deficiency-anemia/ DS00323/DSECTION=complications. Accessed September 5, 2015.

30. Petry NM, Barry D, Pietrzak RH, Wagner JA. Overweight and obesity are associated with psychiatric disorders: results from the National Epidemiologic Survey on Alcohol and Related Conditions. Psychosom Med. 2008;70(3):288-97.

31. Black DW, Shaw M, McCormick B, Allen J. Pathological gambling: relationship to obesity, self-reported chronic medical conditions, poor lifestyle choices, and impaired quality of life. Compr Psychiatry. 2013;54(2):97-104. 32. Finucane MM, Stevens GA, Cowan MJ, et al. National, regional, and global trends in body-mass index since 1980: systematic analysis of health examination surveys and epidemiological studies with 960 country-years and 9.1 million participants. Lancet. 2011;377(9765):557-67.

33. Misra A, Khurana L. Obesity and the metabolic syndrome in developing countries. J Clin Endocrinol Metab. 2008;93(11 Suppl 1):S9-30.

34. Kelly T, Yang W, Chen CS, Reynolds K, He J. Global burden of obesity in 2005 and projections to 2030. Int J Obes (Lond). 2008;32(9):1431-37. 